\section{Alte Eltern}

Prof. Dr. med. Frank-Michael Köhn
$\begin{aligned} & \text { Andrologicum München } \\ & \text { München }\end{aligned}$

43 - Medizinische Risiken später Vaterschaft

47 - Wann ist die Chance auf Mutterschaft vertan?

Wenn der Kinderwunsch immer später verwirklicht wird

\title{
Wann ist Man(n) zu alt fürs Kind?
}

— Risiken und Nachteile der Zeugung von Kindern im höheren Lebensalter sind seit Jahren immer wieder diskutiert worden. Dabei wurden meist die Mütter in den Vordergrund gestellt. Die Frage nach etwaigen Risiken für den Schwangerschaftsverlauf oder die Nachkommen durch die gleichzeitig immer älteren Väter wurde erst relativ spät gestellt. Vor weniger als zehn Jahren begann eine Reihe von Publikationen, sich mit diesem Problemfeld zu beschäftigen, so etwa die Arbeit "Advanced paternal age - how old is too old?" aus dem Jahre 2006 (Bray et al. J Epidemiol Community Health 2006; 60: 851). Ein wesentliches Hindernis bei Erhebungen zum Alter der Väter ist, dass diese Daten gemäß des Grundsatzes „Pater semper incertus est" erst gar nicht erhoben werden oder aus anderen Gründen nicht zur Verfügung stehen. Entsprechende Angaben finden sich z. B. im Statistischen Jahrbuch 2011 des Statistischen Bundesamtes nicht. Als indirekter Hinweis für älter werdende Väter kann nur das Durchschnittsalter lediger Männer zum Zeitpunkt der Hochzeit gelten, das 1985 bei 26,6 Jahren und 2009 bei 33,1 Jahren lag.

Auch im Jahrbuch des Deutschen IVF-Registers sind Informationen zum Alter der Männer spärlich; der Auswertung ist nur zu entnehmen, dass das mittlere Alter der Männer - bei IVF/ICSI ihrer Frauen -

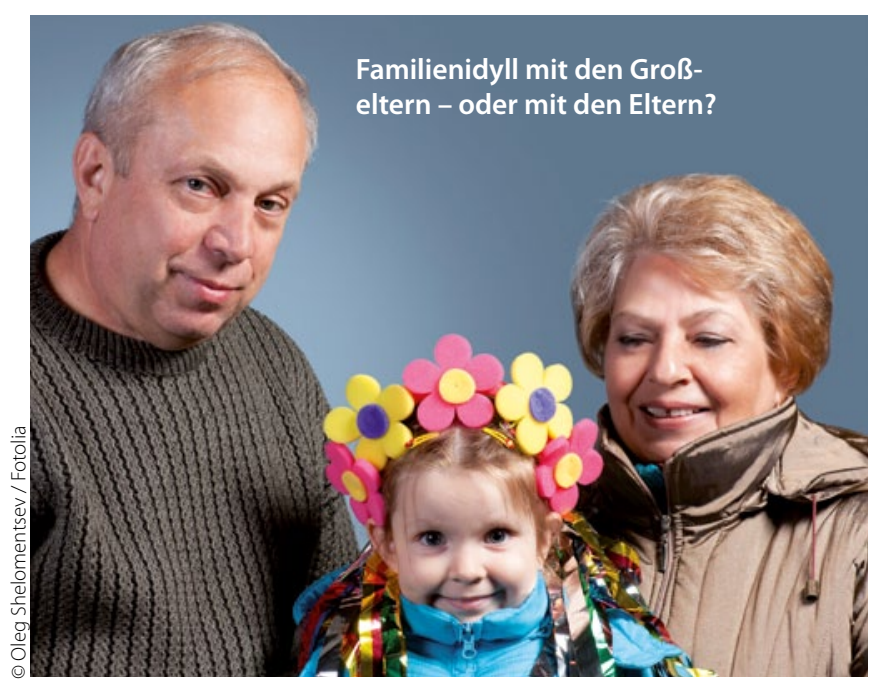

von 1997 bis 2010 von ca. 35 auf 38 Jahre gestiegen ist. Korrelationen zwischen dem Alter der Männer und relevanten Faktoren wie Befruchtungsraten oder Schwangerschaftsverlauf fehlen.

Der gemeinsame Bundesausschuss für Ärzte und Krankenkassen hat in seinen Richtlinien zur künstlichen Befruchtung einen Anspruch auf Leistungen zur künstlichen Befruchtung für Versicherte ausgeschlossen, wenn die Frau das 40. Lebensjahr beendet hat. Das mag nachvollziehbar sein, da ab diesem Alter die Chancen für eine Schwangerschaft deutlich sinken. Für Männer wird eine Altersgrenze von 50 Jahren festgelegt. Gründe hierfür werden nicht angegeben.

Das Problem einer Elternschaft mit zunehmendem Lebensalter ist also vielschichtig:

- Der Vorrang einer beruflichen Qualifikation von Frau und Mann führt zur Verlagerung der Realisierung des Kinderwunsches in spätere Lebensphasen.

- Gesellschaftspolitisch ist ein Anstieg der Geburtenrate erwünscht. - Während Risiken für Mutter und Kind durch eine späte Schwangerschaft letztlich durch die Menopause und den Verlust der Fortpflanzungsfähigkeit eine natürliche Limitierung erfahren (wenn von der bei uns verbotenen Eizellspende und Leihmutterschaft abgesehen wird), kommen Risiken für die Nachkommen älterer Väter durch den langen Erhalt der Fertilität des Mannes stärker zum Tragen.

- Die wissenschaftliche Auseinandersetzung mit Risiken der späten Vaterschaft muss daher intensiviert werden.

- Auch wenn die höhere Lebenserwartung der Eltern den Kindern eine immer längere gemeinsame Lebenszeit mit Vätern und Müttern beschert, sollten dennoch soziologische Aspekte dieser Familien und die Belange dieser Kinder genauer untersucht werden.

Wie sensibel die politische Dimension von Familien mit Kindern ist, lässt derzeit die Diskussion über das Betreuungsgeld erkennen. In Zukunft werden wir uns fragen müssen, ob angesichts der Überalterung unserer Gesellschaft mit entsprechender Belastung der Sozialsysteme die Förderung des späten Kinderwunsches nicht politischgesellschaftlich gewollt ist. Die Medizin wiederum muss fundierte Antworten zu Fragen von Risiken und Früherkennungsmaßnahmen speziell bei älteren Vätern geben. Eine klare Antwort auf die Frage „How old is too old?" darf man dabei für den Mann nicht erwarten! 